\title{
Zero Product of Three Two Level Toeplitz Operators
}

\author{
Matthew Kim ${ }^{1}$, Brian Shon ${ }^{1}$, Albert Cho ${ }^{1}$, Eric Cho $^{1}$, Tedd Jung ${ }^{1} \&$ Omer Mujawar ${ }^{1}$ \\ ${ }^{1}$ Ivy Bridge Academy, Johns Creek, GA, USA \\ Correspondence: Albert Cho, Ivy Bridge Academy, Johns Creek, GA, USA
}

Received: January 13, 2019 Accepted: February 14, 2019 Online Published: February 25, 2019

doi:10.5539/jmr.v11n2p39 URL: https://doi.org/10.5539/jmr.v11n2p39

\begin{abstract}
In this paper we investigate conditions for $T_{f_{1}} T_{f_{2}} T_{f_{3}}-T_{f_{1} f_{2} f_{3}}=0$ where $T_{f_{1}}, T_{f_{2}}$, and $T_{f_{3}}$ are bi-level Toeplitz operators on the Hardy space of bidisk and $f_{1}, f_{2}, f_{3}$ are bounded and measurable complex valued functions on bidisk. We also provide that $T_{f_{1}} T_{f_{2}} T_{f_{3}}$ identical to zero matrix if and only if at least one of $f_{i}$ is identically zero for $1 \leq i \leq 3$.
\end{abstract}

Keywords: Toeplitz operators, Hardy space, bidisk, bi-level Toeplitz matrices

\section{Introduction}

Denote $\mathbb{D}$ be the open unit disk in the complex plane $(\mathbb{C})$ and denote $\mathbb{T}$ be the boundary of it. Then the two subsets of $\mathbb{C}^{2}$, $\mathbb{D}^{2}$ and $\mathbb{T}^{2}$, are simply cartesian product of two copies of $\mathbb{D}$ and $\mathbb{T}$, respectively. Let $H^{2}(\mathbb{T})$ be the usual Hardy space of analytic functions and $P$ be the orthogonal projection on $H^{2}(\mathbb{T})$.

A finite Toeplitz matrix is a matrix which has constant diagonals, namely entries along its diagonals are constant. For any $n \times n$ Toeplitz matrix, $T_{f}$ where $f(z)=\sum_{k=-n+1}^{n-1} \hat{f}_{k}(z) z^{k}$ represents the generating function and $\hat{f}_{k}(z)$ represents Fourier coefficients of $f(z)$

$$
T_{f}=\left[\begin{array}{ccccc}
\hat{f}_{0} & \hat{f}_{-1} & \hat{f}_{-2} & \cdots & \hat{f}_{-n+1} \\
\hat{f}_{1} & \hat{f}_{0} & \hat{f}_{-1} & \cdots & \hat{f}_{-n+2} \\
\vdots & \ddots & \ddots & \ddots & \\
\hat{f}_{-n+1} & \cdots & \cdots & \hat{f}_{1} & \hat{f}_{0}
\end{array}\right]
$$

where the function $\hat{f}(t): \mathbb{T} \rightarrow \mathbb{C}$ and $\mathbb{T}=\{t \in \mathbb{C}:|t|=1\}$. This structure is very interesting in itself for all the rich theoretical properties which it involves, but at the same time it is important for the dramatic impact that it has in applications.

Definition $1 L^{2}(\mathbb{T})$ denotes the Hilbert space of square integrable lebesgue measurable complex valued function on $\mathbb{T}$. For instance, we can define the pointwise operations and inner product as follows:

Let $f_{1}, f_{2} \in L^{2}(\mathbb{T})$. Then

$$
<f_{1}, f_{2}>=\frac{1}{2 \pi} \int_{-\pi}^{\pi} f_{1}(z) \overline{f_{2}(z)} d z, z=e^{i \theta} .
$$

Definition $2 L^{\infty}(\mathbb{T})$ demotes the Banach space of essentially bounded Lebesgue measurable complex valued functions on $\mathbb{T}$. For instance, the pointwise operations and essential norm is defined as follows:

Let $f_{1} \in L^{\infty}(\mathbb{T})$. Then

$$
\left\|f_{1}\right\|_{\infty}=\operatorname{essup}_{|z|=1}\left|f_{1}(z)\right| .
$$

Toeplitz matrices has applications in many different fields. To name a few, time series analysis, filter design, signal processing, etc. Because of its special structure, Toeplitz matrices were analysed by many mathematician. In particular, its algebra. More can be read in (Deepmala, 2014), (Mishra, 2014) and (Mishra, 2017). For instance, the product of two Toeplitz operators may not be Toeplitz again. It is easy to check that when a Toeplitz operator is the zero operator, then its corresponding symbol is zero and vice versa. It is also known that: the product of two such operators is identical to zero if and only if the symbol of one of them is zero (Brown \& Halmos, 1963/1964). This result was later generalized for products of many Toeplitz operators, (Aleman \& Vukotic, 2009). In (Ding, Sun \& Zheng, 2012), Theorem 1.5 gives us necessary and sufficient condition for the product of bi-level Toeplitz operators Hardy space of the bidisk. In this paper we consider the following: Can we find a necessary and sufficient conditions to make the product of three bi-level infinite Toeplitz matrices is a Toeplitz matrix.? This paper is organized as follows: In section 1, we state and give the proof of some known results. In section 2, we give two main results: (1) We find necessary and sufficient conditions for when the 
product of three bi-level Toeplitz operators is again Toeplitz (2) We prove that, $T_{f_{1}} T_{f_{2}} T_{f_{3}}$ identical to zero matrix if and only if at least one of $f_{i}$ is identically zero for $1 \leq i \leq 3$.

We would like to recall some known results regarding the zero product of one level Toeplitz operators.

Lemma 1 Suppose $T_{f_{1}}$ and $T_{f_{2}}$ are classical Toeplitz operators where $f_{1}(z)$ and $f_{2}(z)$ are bounded and measurable complex valued functions on the unit circle. Let $A=T_{f_{1}} T_{f_{2}}$ and let $\left(a_{i j}\right)$ represent the $(i, j)$-entry of the matrix of $A$. Let the Fourier expansions of $f_{1}(z)$ and $f_{2}(z)$ be $f_{1}(z)=\sum_{k=-\infty}^{\infty}\left(\hat{f_{1}}\right) k(z) z^{k}$ and $f_{2}(z)=\sum_{k=-\infty}^{\infty}\left(\hat{f_{2}}\right)_{k}(z) z^{k}$, where $\left(\hat{f_{1}}\right)_{k}(z)$ and $\left(\hat{f_{2}}\right)_{k}(z)$ are the Fourier coefficients of $f_{1}(z)$ and $f_{2}(z)$. Then

$$
a_{i+1, j+1}=a_{i j}+\left(\hat{f}_{1}\right)_{i+1}\left(\hat{f}_{2}\right)_{-j-1}
$$

whenever $i, j \geq 0$.

Proof. We can $(i, j)-$ entry of the matrix as follows

$$
a_{i j}=\sum_{k=0}^{\infty}\left(\hat{f}_{1}\right)_{i-k}\left(\hat{f}_{2}\right)_{k-j} .
$$

Then by some simple manipulations of the sum, we have the following

$$
\begin{aligned}
a_{i+1, j+1} & =\sum_{k=0}^{\infty}\left(\hat{f}_{1}\right)_{i+1-k}\left(\hat{f}_{2}\right)_{k-j-1} \\
& =\left(\hat{f}_{1}\right)_{i+1}\left(\hat{f}_{2}\right)_{-j-1}+\sum_{k=1}^{\infty}\left(\hat{f}_{1}\right)_{i+1-k}\left(\hat{f}_{2}\right)_{k-j-1} \\
& =\left(\hat{f}_{1}\right)_{i+1}\left(\hat{f}_{2}\right)_{-j-1}+\sum_{k=0}^{\infty}\left(\hat{f}_{2}\right)_{i-k}\left(\hat{f}_{2}\right)_{k-j} \\
& =\left(\hat{f}_{1}\right)_{i+1}\left(\hat{f}_{2}\right)_{-j-1}+a_{i j} .
\end{aligned}
$$

The following result is by (Brown \& Halmos, 1963/1964).

\section{Theorem 1}

Let $f_{1}(z)$ and $f_{2}(z)$ be bounded and measurable complex valued functions. Then $T_{f_{1}} T_{f_{2}}=T_{f_{1} f_{2}}$ if and only if one of the following holds

(i) $f_{1}$ and $f_{2}$ are analytic

(ii) $f_{1}$ and $f_{2}$ are co-analytic

(iii) There exist constants $c_{1}$ and $c_{2}$ with $\left|c_{1}\right|+\left|c_{2}\right| \neq 0$ such that $c_{1} f_{1}+c_{2} f_{2}$ is constant.

Proof.

Suppose $f_{2}(z)$ is analytic. Then

$$
T_{f_{1}} T_{f_{2}} g=T_{f_{1}}\left(f_{2} g\right)=P\left(f_{1} f_{2} g\right)=T_{f_{1} f_{2}} g,
$$

where $P$ is an orthogonal projection on $H^{2}(\mathbb{T})$ and for all $g(z) \in H^{2}$, and thus $T_{f_{1}} T_{f_{2}}=T_{f_{1} f_{2}}$. Next suppose $f_{1}(z)$ is co-analytic. Then $\bar{f}_{1}(z)$ is analytic, and thus

$$
T_{f_{1}} T_{f_{2}}=\left(T_{\bar{f}_{2}} T_{\bar{f}_{1}}\right)^{*}=T_{\bar{f}_{1} \bar{f}_{2}}^{*}=T_{f_{1} f_{2}} .
$$

The following result is by (Riesz \& Riesz, 1916).

\section{Theorem 2}

Let $F$ be analytic on $\mathbb{D}$ and $L^{1}$-bounded, i.e, $F \in H^{1}(\mathbb{D})$. Assume that $F \neq 0$ and set $f=\lim _{r \rightarrow 1^{-}} F_{r}$. Then $\log |f| \in L^{1}(\mathbb{T})$. In particular, $f$ does not vanish on a set of positive measure.

The following result is by (Brown \& Halmos, 1963/1964). 


\section{Theorem 3}

Let $f_{1}(z)$ and $f_{2}(z)$ be bounded measurable complex valued functions on the unit disk. Then $T_{f_{1}} T_{f_{2}}$ is identical to zero operator if and only if $f_{1}(z)=0$ or $f_{2}(z)=0$.

Proof.

Suppose either $f_{1}(z)=0$ or $f_{2}(z)=0$, then we have either $T_{f_{1}}=0$ or $T_{f_{2}}=0$, where 0 is zero operator matrix. Then, it follows that $T_{f_{1}} T_{f_{2}}=0$. Next suppose that $T_{f_{1}} T_{f_{2}}=0$. Then, since 0 is a Toeplitz operator, it follows from Theorem 1 that either $f_{1}(z)$ is co-analytic or $f_{2}(z)$ is analytic and that $f_{1}(z) f_{2}(z)=0$. By Theorem 2 , since a non-zero analytic function, cannot vanish on a set of positive measure, it follows that if $f_{1}(z)$ is co-analytic, then $f_{1}(z)=0$, and if $f_{1}(z)$ is analytic, then $f_{1}(z)=0$.

\section{Main Results}

Theorem 4 Suppose

$$
f_{1}\left(z_{1}, z_{2}\right)=\sum_{(i, j) \in \mathbb{Z} \times \mathbb{Z}} \hat{f}_{1}(i, j) z_{1}^{i} z_{2}^{j}, \quad f_{2}\left(z_{1}, z_{2}\right)=\sum_{(i, j) \in \mathbb{Z} \times \mathbb{Z}} \hat{f}_{2}(i, j) z_{1}^{i} z_{2}^{j}
$$

and

$$
f_{3}\left(z_{1}, z_{2}\right)=\sum_{(i, j) \in \mathbb{Z} \times \mathbb{Z}} \hat{f}_{3}(i, j) z_{1}^{i} z_{2}^{j}
$$

where $f_{1}\left(z_{1}, z_{2}\right), f_{2}\left(z_{1}, z_{2}\right)$ and $f_{3}\left(z_{1}, z_{2}\right)$ are $L^{\infty}\left(\mathbb{T}^{2}\right)$ functions, and $\hat{f}_{1}(i, j), \hat{f}_{2}(i, j), \hat{f}_{3}(i, j)$ are the Fourier coefficients of $f_{1}\left(z_{1}, z_{2}\right), f_{2}\left(z_{1}, z_{2}\right), f_{3}\left(z_{1}, z_{2}\right)$, respectively. Then we have

$T_{f_{1}} T_{f_{2}} T_{f_{3}}=T_{f_{1} f_{2} f_{3}}$ is Toeplitz if and only if one of the following conditions holds for almost all $a, b \in \mathbb{T}$.

(i) $f_{1}\left(z_{1}, a\right), f_{2}\left(z_{1}, a\right)$ and $f_{3}\left(z_{1}, a\right)$ are all analytic in variable $z_{1}$ on $\mathbb{T}$.

(ii) $f_{1}\left(z_{1}, a\right), f_{2}\left(z_{1}, a\right)$ and $f_{3}\left(z_{1}, a\right)$ are co-analytic in variable $z_{1}$ on $\mathbb{T}$.

(iii) $f_{1}\left(b, z_{2}\right), f_{2}\left(b, z_{2}\right)$ and $f_{3}\left(b, z_{2}\right)$ are all analytic in variable $z_{2}$ on $\mathbb{T}$.

(iv) $f_{1}\left(b, z_{2}\right), f_{2}\left(b, z_{2}\right)$ and $f_{3}\left(b, z_{2}\right)$ are all co-analytic in variable $z_{2}$ on $\mathbb{T}$.

(v) $f_{1}\left(z_{1}, a\right), f_{3}\left(z_{1}, a\right)$ are analytic in variable $z_{1}$ and $f_{2}\left(z_{1}, a\right)$ is co-analytic in variable $z_{1}$ on $\mathbb{T}$.

(vi) $f_{1}\left(b, z_{2}\right), f_{3}\left(b, z_{2}\right)$ are analytic in variable $z_{2}$ and $f_{2}\left(b, z_{2}\right)$ is co-analytic in variable $z_{2}$ on $\mathbb{T}$.

Proof. Suppose that $T_{f_{1}} T_{f_{2}} T_{f_{3}}=T_{f_{1} f_{2} f_{3}}$. Let $T_{f_{1}} T_{f_{2}}=T_{h}$. Then using (Ding, Sun and Zheng, 2012), in particular Theorem $1.5, T_{h}$ is Toepltiz and we have that $f_{1}$ and $f_{2}$ are both analytic in variable $z_{1}$ on $\mathbb{D}$ or $f_{1}$ and $f_{2}$ are both co-analytic in variable $z_{1}$ on $\mathbb{D}$ and $f_{1}$ and $f_{2}$ are both analytic in variable $z_{2}$ on $\mathbb{D}$ or $f_{1}$ and $f_{2}$ are both co-analytic in variable $z_{2}$ on $\mathbb{D}$.

To prove the converse,we will prove (i),(ii) and the proof of other cases are similar. Now suppose $f_{1}\left(z_{1}, a\right), f_{2}\left(z_{1}, a\right)$ and $f_{3}\left(z_{1}, a\right)$ are all analytic in variable $z_{1}$ on $\mathbb{T}$, then we will prove that $T_{h} T_{f_{3}}$ is Toeplitz. Let $i, j, l \in \mathbb{N}_{0}^{2}$. Write

$$
\left(T_{h} T_{f_{3}}\right)_{(i, j)}=\sum_{k \in \mathbb{N}_{0}^{2}} \hat{h}_{(i-k)} \hat{f}_{3(k-j)}
$$

Since $k_{1}-i_{1} \geq 0$ and $k_{2}-i_{2} \geq 0$, then the equation (2) is changed to the following form:

$$
\left(T_{h} T_{f_{3}}\right)_{(i, j)}=\sum_{k \in\left(\left(i_{1}, 0\right)+\mathbb{N}_{0}^{2} \cap\left(j_{2}, 0\right)+\mathbb{N}_{0}^{2}\right)} \hat{h}_{i-k} \hat{f}_{3(k-j)}
$$

Then we can write

$$
\left(T_{h} T_{f_{3}}\right)_{(i+l, j+l)}=\sum_{k \in\left(\left(i_{1}+l_{1}, 0\right)+\mathbb{N}_{0}^{2} \cap\left(i_{2}+l_{2}, 0\right)+\mathbb{N}_{0}^{2}\right)} \hat{h}_{i-k+l} \hat{f}_{3(k-j-l)}
$$

Substitute $\tilde{k}$ by $k-l$, the we get $i_{1} \leq \tilde{k}_{1}$ and $i_{2} \leq \tilde{k}_{2}$. So the equation (4) changed to the following form:

$$
\left(T_{h} T_{f_{3}}\right)_{(i+l, j+l)}=\sum_{\tilde{k} \in\left(i_{1}, i_{2}\right)+\mathbb{N}_{0}^{2}} \hat{h}_{i-\tilde{k}} \hat{f}_{3(\tilde{k}-j)}
$$


Hence we showed that the equations (3) and (5) are equal.

Now suppose that $f_{1}\left(z_{1}, a\right), f_{2}\left(z_{1}, a\right)$ and $f_{3}\left(z_{1}, a\right)$ are co-analytic in variable $z_{1}$ on $\mathbb{T}$. Then we can the following:

$$
\left(T_{h} T_{f_{3}}\right)_{(i, j)}=\sum_{k \in \mathbb{N}_{0}^{2}} \hat{h}_{i-k} \hat{f}_{3(k-j)}
$$

Since $k_{1}-i_{1} \geq 0$ and $k_{2}-j_{2} \leq 0$, then the equation (6) is changed to the following form:

$$
\left(T_{h} T_{f_{3}}\right)_{(i, j)}=\sum_{k \in\left(\left(i_{1}, 0\right)+\mathbb{N}_{0}^{2} \cap\left(0, j_{2}\right)+\mathbb{N}_{0}^{2}\right)} \hat{h}_{i-k} \hat{f}_{3(k-j)}
$$

Next we can write

$$
\left(T_{h} T_{f_{3}}\right)_{(i+l, j+l)}=\sum_{k \in\left(\left(j_{1}+l_{1}, 0\right)+\mathbb{N}_{0}^{2} \cap\left(0, j_{2}+l_{2}\right)+\mathbb{N}_{0}^{2}\right)} \hat{h}_{i-k+l} \hat{f}_{3(k-j-l)}
$$

Letting $\tilde{k}=k-l$, we get $i_{1} \leq \tilde{k}_{1}, \tilde{k}_{2} \leq j_{2}, \tilde{k}_{2} \leq-l_{2}$ and $-l_{1} \leq \tilde{k}_{1}$. This implies that $\tilde{k} \in\left(\left(i_{1},-l_{2}\right)+\mathbb{N}_{0}^{2} \cap\left(-i_{2}, j_{2}\right)+\mathbb{N}_{0}^{2}\right) \cap$ $\left(\left(i_{1}, 0\right)+\mathbb{N}_{0}^{2} \cap\left(0, j_{2}\right)+\mathbb{N}_{0}^{2}\right)$. Then the equation (8) is changed to the following form:

$$
\left(T_{h} T_{f_{3}}\right)_{(i+l, j+l)}=\sum_{\tilde{k} \in \Lambda} \hat{h}_{i-\tilde{k}} \hat{f}_{3(\tilde{k}-j)} .
$$

Now we can conclude that equations (7) and (9) are equal.

Theorem $5 T_{f_{1}} T_{f_{2}} T_{f_{3}}$ is identically zero matrix if and only if at least one of $f_{i}=0$ for $1 \leq i \leq 3$.

Proof. First suppose one of $f_{i}$ is zero. Then it is clear that $T_{f_{i}}=0$, where 0 is the zero matrix. For the converse, now suppose $T_{f_{1}} T_{f_{2}} T_{f_{3}}=0$. Then, since 0 is a Toeplitz operator, it follows from Theorem 4 that one of the six cases hold and that $f_{1} f_{2} f_{3}=0$. In the case where $f_{1}$ is co-analytic in $z_{1}$ and $z_{2}$ or $f_{2}$ and $f_{3}$ is analytic in $z_{1}$ and $z_{2}$, then by Theorem of (F. and M. Riesz, 2016), a non-zero analytic function cannot vanish on a set of positive measure, this implies that if $f_{1}$ is co-analytic in $z_{1}$ and $z_{2}$, then $f_{2} f_{3}=0$ and if $f_{3}$ is analytic in $z_{1}$ and $z_{2}$, then $f_{1} f_{2}=0$. In the case where one of the $f_{i}$ is co-analytic in $z_{1}$ and the others are analytic in $z_{2}$, we have that $f_{1} f_{2} f_{3}=0$ a.e on $\mathbb{T}^{2}$. Let $A \times B \subseteq \mathbb{T} \times \mathbb{T}$ be the zero set of $f_{1}$ and $A$ have positive measure in $\mathbb{T}$. Since $f_{1}$ is co-analytic in $z_{1}$ and $f_{1} \in L_{\infty}\left(\mathbb{T}^{2}\right)$, then for each fixed $z_{2} \in \mathbb{T}$, we have that $f_{1} \in H^{2}(\mathbb{T})$. Thus $f_{1}=0$ a.e for $z_{1} \in \mathbb{T}$ and $z_{2} \in B$, i.e, $A=B$ and hence $f_{1}=0$ on $\mathbb{T} \times B$. If the set $B$ has full measure in $\mathbb{T}$, then $f_{1}=0$ on $\mathbb{T}^{2}$. However, assume that $B$ is not of full measure in $\mathbb{T}$, then $f_{2}=0$ on $\mathbb{T}^{2}-\mathbb{T} \times B=\mathbb{T} \times(\mathbb{T}-B)$ and $\mathbb{T}-B$ has positive measure. But $f_{2}$ is analytic in $z_{2}$ and for fixed $z_{1} \in \mathbb{T}$, we have $f_{1} \in H^{2}(\mathbb{T})$. Note that this implies $f_{2}=0$ a.e for $z_{2} \in \mathbb{T}$. Thus $\mathbb{T}-B=\mathbb{T}$, i.e, $f_{2}=0$ on $\mathbb{T}^{2}$. Same argument can repeated for any $f_{i}$, where $1 \leq i \leq 3$.

\section{Conclusion}

In this manuscript, we consider the following question: Can we find a necessary and sufficient conditions to make the product of three bi-level infinite Toeplitz matrices again a Toeplitz matrix.? This question is just a natural extension of a well known a result by (Brown \& Halmos, 1963/1964) in the case one level Toepltiz operators. In this paper, we extended the result by (Brown \& Halmos, 1963/1964) to the case when three bi-level Toeplitz operator are given. Then we provide two main results: (1) we find necessary and sufficient conditions for when the product of three bi-level Toeplitz operators is again Toeplitz (2) we prove that, $T_{f_{1}} T_{f_{2}} T_{f_{3}}$ identical to zero matrix if and only if at least one of $f_{i}$ is identically zero for $1 \leq i \leq 3$.

\section{Acknowledgements}

We would like thank to reviewers for their valuable suggestions.

\section{References}

Aleman, A., \& Vukotic, D. (2009). Zero product of Toeplitz operators. Duke Math. J., 148(3), 373-403. https://doi.org/10.1215/00127094-2009-029

Brown, A., \& Halmos, P. R. (1963/1974). Algebraic properties of Toeplitz operators. J. Reine Angew. Math. 213 , 89-102.

Ding, X., Sun, S., \& Zheng, D. (2012). Commuting Toeplitz operators on the bidisk. J. Funct. Anal., 263(11), 3333-3357. https://doi.org/10.1016/j.jfa.2012.08.005

Riesz, F., \& Riesz, M. (1916). Uber die randwerte einer analytischen funktion. Quatrieme Congres des Mathematiciens Scandinaves, Stockholm, 27-44. 
Deepmala. (2014). A Study on Fixed Point Theorems for Nonlinear Contractions and its Application. Ph.D. Thesis, Pt. Ravishankar Shukla University, Raipur 492010, Chhatisgarh, India.

Mishra, L. N. (2017). On the existence and behavior of solutions to some nonlinear integral equations with Applications. Ph.D Thesis, National Institute of Technology, Silchar 788010, Assam, India.

Mishra, L. N., Mishra, V. N., \& Khatri, K. (2014). On The Trigonometric approximation of signals belonging to generalized weighted Lipschitz $W\left(L^{r}, \xi t\right)(r \geq 1)$ class by matrix $\left(C^{1} \cdot N_{p}\right)$ Operator of conjugate series of its Fourier series. Applied Mathematics and Computation, 237, 252-263.

\section{Copyrights}

Copyright for this article is retained by the author(s), with first publication rights granted to the journal.

This is an open-access article distributed under the terms and conditions of the Creative Commons Attribution license (http://creativecommons.org/licenses/by/4.0/). 